\title{
Statistical properties of earthquakes clustering
}

\author{
A. Vecchio ${ }^{1}$, V. Carbone ${ }^{1}$, L. Sorriso-Valvo ${ }^{2}$, C. De Rose ${ }^{1}$, I. Guerra ${ }^{1}$, and P. Harabaglia ${ }^{3}$ \\ ${ }^{1}$ Dipartimento di Fisica, Università della Calabria, Rende (CS), Italy \\ ${ }^{2}$ LICRYL-INFM/CNR, ponte P. Bucci, cubo 31 C, 87036 Rende (CS), Italy \\ ${ }^{3}$ Dipartimento di Strutture, Geotecnica e Geologia Applicata all'Ingegneria, Università della Basilicata, Potenza, Italy
}

Received: 21 February 2008 - Revised: 4 March 2008 - Accepted: 17 March 2008 - Published: 16 April 2008

\begin{abstract}
Often in nature the temporal distribution of inhomogeneous stochastic point processes can be modeled as a realization of renewal Poisson processes with a variable rate. Here we investigate one of the classical examples, namely, the temporal distribution of earthquakes. We show that this process strongly departs from a Poisson statistics for both catalogue and sequence data sets. This indicate the presence of correlations in the system probably related to the stressing perturbation characterizing the seismicity in the area under analysis. As shown by this analysis, the catalogues, at variance with sequences, show common statistical properties.
\end{abstract}

\section{Introduction}

Seismic events are very complex spatio-temporal phenomena (see e.g. Turcotte, 1993; Sornette, 1999; Bak et al., 2002, and references therein). Seismic catalogues, reporting informations about spatio-temporal distribution of the main shocks, are nowaday available for many seismic areas in the world. Very often major events mark the beginning of a series of earthquakes (aftershocks) whose frequency and energy are meanly decreasing in time down to the background level of activity. In some cases, main shocks are also preceded by minor earthquakes (foreshocks). Earthquakes sequence catalogues, restricted to single main shocks-aftershocks series, show that both foreshocks and aftershocks are generally well space-correlated with sources being distributed along or near the same fractured surface of the main shock. Seismic catalogues and sequences are complete only above a threshold magnitude that depends primarily on the characteristics and geometry of the observing instrumental network.

Earthquakes are in general statistically characterized by three empirical laws: i) calm intervals $t$ between successive aftershocks exhibit a power law distribution $t^{-\alpha}$ (with $\alpha>1$ ), commonly explained in the framework of the em-

Correspondence to: A. Vecchio

(vecchio@fis.unical.it) pirical Omori law (Omori, 1895); ii) the logarithm of the number of earthquakes with magnitude above a given threshold $m$ behaves as $-b m$ with $b$ constant (Gutenberg-Richter's law, Gutenberg and Richter, 1944); iii) the epicentres within a seismic region follow a fractal distribution (Turcotte, 1993). The Omori law describes the frequency of occurrence of the aftershocks that releases an energy considerably greater than the mean of the similar events that are randomly recorded in a given geological domain.

Analyzing a catalogue of Californian seismic events in the period 1984-2000 Bak et al. (2002) found that the different statistics seems to be unified in a single scaling law. This is seen considering both spatial and time dependence. In particular authors found no operational difference that might lead to distinguish between aftershocks and major earthquakes. Starting from the Bak results the unified scaling law was developed in some other works (Corral, 2003, 2004; Saichev and Sornette, 1895).

Later articles (Davidsen and Goltz, 2004; Carbone et al., 2005) lead to the conclusions that the universality claimed by Bak et al. (2002) is not valid. In particular Carbone et al. (2005), selecting and analyzing some different sequences of aftershocks independently from the usual catalogues, found that the probability distribution for the interevents times is well described by Lévy functions. On the base of this relation it results that the seismic events occurrence pattern significantly departs from a Poissonian distribution. While this results were confirmed in some other works (see e.g. Shcherbakov et al., 2004, 2005) the same conclusions are not so evident when general seismic catalogues are analyzed. Thus more refined statistical analysis are requested to quantify the clustering of events in seismic sequences and to characterize the time distribution of main shocks.

Studies and modelizations of the inter-events times distribution were perfomed following different methods (Shcherbakov et al., 2004, 2005; Lippiello et al., 2007). In this paper we apply a method introduced some years ago in cosmology (Bi et al., 1989) and used more recently in the contex of solar flares (Lepreti et al., 2001) and paleomagnetic reversal (Carbone et al., 2006; Sorriso-Valvo et al., 2007), to

Published by Copernicus Publications on behalf of the European Geosciences Union and the American Geophysical Union. 

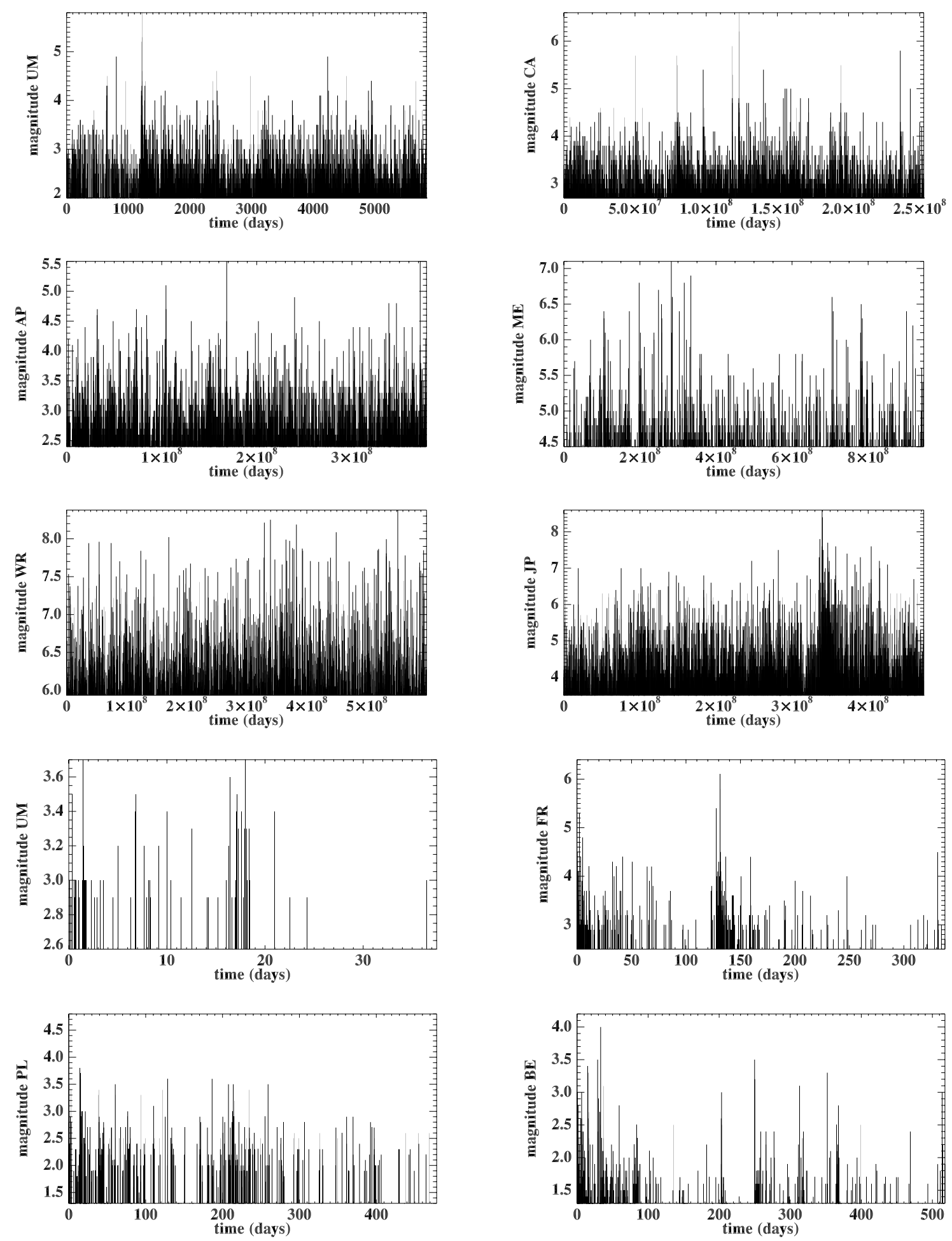

Fig. 1. The ten data set used for the analysis. The upper block corresponds to the catalogue data sets, the lower one refers to the sequences. 

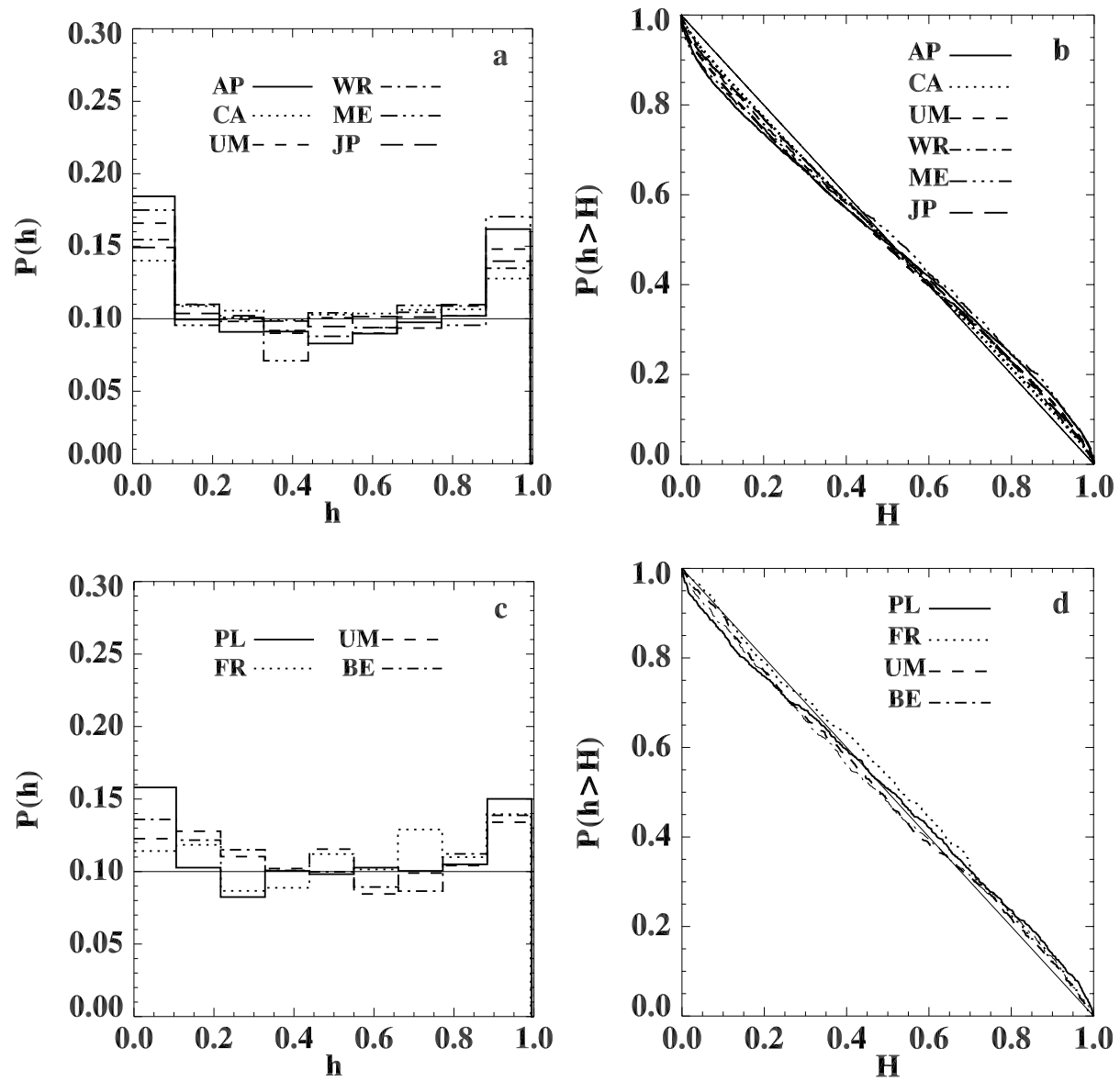

Fig. 2. Probability distribution functions $P(h)$ of the stochastic variable $h$ and corresponding surviving functions $P(h \geq H)$ for all the empirical datasets (seismic catalogues panels a, b and aftershocks sequences panels $\mathrm{c}, \mathrm{d}$ ). The theoretical probability expected under a Poisson statistics is also shown.

characterize the time clustering of major earthquakes and aftershocks.

\section{Data analysis and results}

Looking at the earthquakes as a temporal point process we can consider the sequence of time intervals between two successive events $\delta t$ (waiting times or interevents times) as a function of the earthquake occurrence time $t$. We can test whether the waiting time sequence is consistent with a time varying Poisson process without any a priori assumption or, instead, correlations are present in the system leading to clustering of the events. Starting from the waiting times sequence we introduce the stochastic variable $h$ that simply represents the suitably normalized local time between earthquakes

$h_{i}\left(\delta t_{i}, \delta \tau_{i}\right)=\frac{2 \delta t_{i}}{2 \delta t_{i}+\delta \tau_{i}}$,

where $\delta t_{i}$ and $\delta \tau_{i}$ are defined as

$\delta t_{i}=\min \left\{t_{i+1}-t_{i}, t_{i}-t_{i-1}\right\}$ $\delta \tau_{i}= \begin{cases}t_{i-1}-t_{i-2} & \text { if } \delta t_{i}=t_{i}-t_{i-1} \\ t_{i+2}-t_{i+1} & \text { if } \delta t_{i}=t_{i+1}-t_{i}\end{cases}$

$\delta t_{i}$ and $\delta \tau_{i}$ are then two interevents times following or preceding a given earthquake at $t_{i}$.

Let us suppose that $\delta t_{i}$ and $\delta \tau_{i}$ are independently distributed with probability densities given by $p\left(\delta t_{i}\right)=2 \lambda_{i} \exp \left(-2 \lambda_{i} \delta t_{i}\right)$ and $p\left(\delta \tau_{i}\right)=\lambda_{i} \exp \left(-\lambda_{i} \delta \tau_{i}\right)$. In this case it can be easily shown that the distribution of $h$ does not depend on the local event rate $\lambda_{i}$.

Indeed, defining the surviving function of the probability distribution function

$$
\begin{array}{r}
P(h \geq H)=\int_{H}^{\infty} P(h) d h= \\
=\int_{0}^{\infty} d x 2 \lambda e^{-2 \lambda x} \int_{0}^{g(x, H)} d y \lambda e^{-\lambda x}=1-H
\end{array}
$$

where $P(h)$ is the probability distribution function of $h$ and $g(x, h)=2 x\left[\frac{1}{H}-1\right]$. This means that, under hypothesis that the events are locally distributed according to Poisson 
Table 1. Probabilities calculated using the Kolmogorov-Smirnov test. Small values of probability show that the cumulative distribution function of data is significantly different from the uniform one.

\begin{tabular}{lcr}
\hline & events number & probability \\
\hline \multicolumn{3}{c}{ Catalogues } \\
AP & 4250 & $10^{-21}$ \\
CA & 23886 & $10^{-19}$ \\
UM & 12562 & $10^{-35}$ \\
MO & 2541 & $10^{-5}$ \\
ME & 1313 & $10^{-5}$ \\
JP & 2296 & $10^{-10}$ \\
\hline & & \\
\hline & Sequences \\
PL & 890 & $7 \cdot 10^{-3}$ \\
FR & 477 & $4 \cdot 10^{-3}$ \\
UM & 1619 & $10^{-1}$ \\
BE & 1056 & $4 \cdot 10^{-2}$ \\
\hline
\end{tabular}

statistics, the variable $h$ is uniformly distributed between 0 and 1 . In a process where $\delta \tau_{i}$ are sistematically smaller than $2 \delta t_{i}$, clusters are present and the average value of $h$ is greater than $1 / 2$. On the contrary, when the process is characterized by the presence of voids, the average value of $h$ is less than $1 / 2$. Thus, the test can give information on the presence of correlations in the system, and allows a quantitative description of clustering properties of the events.

We apply the test decribed above on several different data sets from both general earthquakes catalogues and aftershocks sequences (see Fig. 1). Regarding the sequences, the analized data are relative to Italy, where we have a knowledge of the tectonic settings of the areas and their seismic history. In detail, the general catalogue of the peninsular Italy was extracted from the "ING-GNDT Gruppo di lavoro Catalogo Strumentale" (2001) catalog and refers to the period 1985-1996 period, at latitudes between 37.5 and $44.5 \mathrm{~N}$ (set AP). We also selected the sequence localized on the western slope of the Pollino chain at the border between Calabria and Basilicata (southern Italy) during the period 1998-1999 (set PL (Guerra et al., 2005)) and the Friuli Catalogues (northeast Italy) during about one year from 1976 to 1977 (set FR, curtesy by D. Slejko, INOGS-Trieste). The sequence indicated as BE, covering about one year from 1997 to 1998 , is extracted by the CSI catalogue and refers to the Benevento area (south Italy). For many seismological aspects, e.g. Antonioli et al. (2005), the seismic area of Umbria-Marche (central Italy) and surroundings is of particular interest. For this area, the catalog in the period 1981-1996 at latitudes 41.5$44.5 \mathrm{~N}$ and the sequence in the period 1997-1998 were analyzed (sets UM).

To verify the reliability of our analysis we enlarge the data sets with the catalog of Southern California (set CA, from

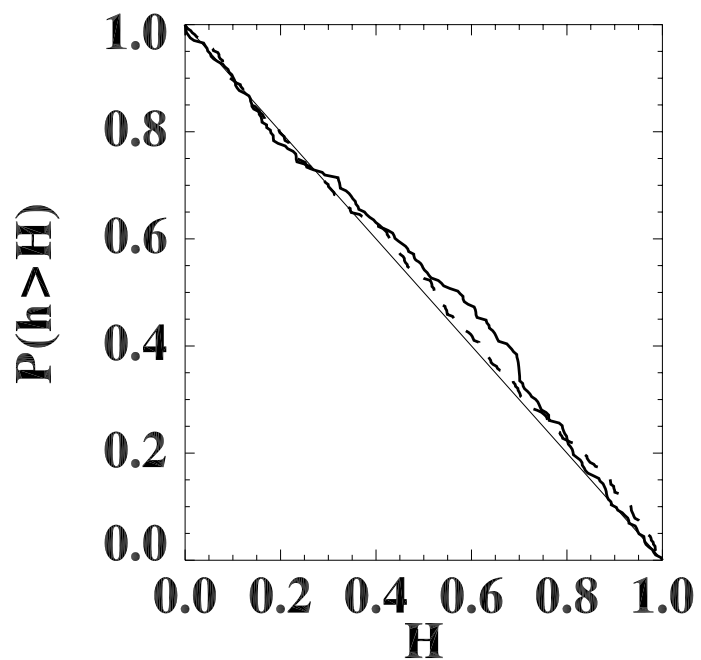

Fig. 3. Surviving functions $P(h \geq H)$ for the two data sets extracted from the FR sequence. The theoretical probability expected under a Poisson statistics is also shown.

Southern California Eartquake data Center, 1984-2002), the NIED Catalogue of Japan and surroundings (set JP, from National Research Institute for Earth Science and Disaster Prevention of Japan (NIED) seismic moment tensor (MT) catalogue, 1984-1998), the Catalogue of Eastern Mediteranean based on the NEIC data locations (set ME, from USGS National Earthquake Information Center, 1973-2002) and a global catalogue based on Harvard CMT solutions (set WR, from Centroid Moment Tensor catalog-Harvard (CMT), 1984-2002).

In Fig. 2 the probability distribution functions $P(h)$ are shown for all the datasets from earthquakes catalogues (panel a) and aftershocks sequences (panel c). In the same figure the corresponding surviving functions $P(h \geq H)$ are represented in panels $b, d$ for the general catalogues and sequences data sets respectively. A significant deviation from the uniform distribution can clearly be observed for all the datasets. A Kolmogorov-Smirnov (KS) test applied to the distributions confirms that the uniform distribution is not compatible with the data, and must be rejected (the significance level of the KS test being smaller than $0.5 \%$ for all datasets, see Table 1). Starting from the general catalogue data sets, we analize more in detail the properties of the probability functions $P(h)$ and the correspondig surviving functions $P(h \geq H)$. The $P(h)$ are characterized by a typical shape, being roughly constant for $0<h<1$ and showing two peaks around $h \approx 0$ and $h \approx 1$. As a consequence, the situation in which long waiting times are followed by short waiting times (or viceversa, since this analysis is not able to distinguish the two cases) is highly frequent in the catalogue data. Moreover, this indicates that clusters are not present in the earthquakes waiting times extract from catalogues. This is probably due to the short temporal span (few decades) of catalogues with respect to the 
characteristic clustering time. In fact a qualitative analysis of CPTI (Parametric Catalogue of the Italian earthquakes, 217 B.C.-2002) shows a succession of intervals of low and high seismic activity on time scales of the order of 100 years.

As for the $P(h)$, the $P(h \geq H)$ behaviors are similar for all the data sets. However the $P(h \geq H)$ departure from the uniform theoretical surviving function is less evident for the California data set with respect the other curves (Fig. 2b). This result is in agreement with Carbone et al. (2005), where California catalogue was shown to own peculiar statistical properties in comparison with other catalogues.

Now we turn our attention to the data sets extracted from the seismic sequences. While the PL and BE $P(h)$ show a behavior similar to the catalogues density functions, with peaks at $h \approx 0$ and $h \approx 1$, the FR and UM $P(h)$ shapes appear quite different. In these cases the $P(h)$ departure from the uniform distribution takes place in a more regular way (Fig. 2, panel c) with a progressive increase of $P(h)$ away from $h \approx 0$ and $h \approx 1$. This is an indication of presence of clusters. The clusters in the UM and FR sequences are evident also looking at the corresponding data sets (see Fig. 1). On the contrary the temporal behavior of the PL and BE records appear to be more similar to the catalogues ones. The peculiar characteristic common to the UM and FR data sets is the presence of multiple main events into the analyzed sequence. In fact, the UM data set shows six major earthquakes with magnitudes greater than 5 and two earthquakes with magnitude above the same threshold can be recognized in the FR sequence. The major events, together with the corresponding aftershocks, form, in fact, the detected clusters as could be expected.

The $P(h \geq H)$ shapes confirm the sequence properties deduced from the $P(h)$ and put in evidence the presence of clusters in the FR and UM data sets. In particular, for the FR data set the surviving function is mostly above $h>1 / 2$.

The clustering properties of the FR data set can be put in evidence analyzing separately the aftershocks sequences following the two main events. As can be expected the surviving functions (see Fig. 3) show only values $h>1 / 2$ indicating a clustering.

The same beviour is not evident in the $P(h \geq H)$ calculated for the UM record. The presence of six major events mixes the clustering and declustering effect. The UM surviving function, however, mantain a peculiar shape.

\section{Conclusions}

In conclusion, we investigated the statistics of persistence times between earthquakes. We applied a statistical test, showing that waiting times between earthquakes stem from an underlying process that is far from being locally Poissonian, indicating the presence of correlations in the system. These could be induced by some mechanism triggering the seismicity (Godano and Pingue, 2005; Tosi, 2004). For the UM sequence this can be identified in the pore-fluid flow (see Antonioli et al., 2005 and references therein). The properties of the catalogues data sets seem to be very similar among them with a slight but significant deviation for California. The non-poissonian character is mainly due to alternating long-short intervals between successive records. The sequences data sets, instead, show a more regular deviation from a Poisson process. In particular, two sequences data show clustering probably related to the peculiar aspects of the stress field in the corresponding areas.

Edited by: R. Vautard

Reviewed by: two anonymous referees

\section{References}

Antonioli, A., Piccinini, D., Chiaraluce, L., and Cocco, M.: Fluid flow and seismicity pattern: Evidence from the 1997 UmbriaMarche (central Italy) seismic sequence, Geophys. Res. Lett., 32, L10311, doi:10.1029/2004GL022256, 2005.

Bak, P., Christensen, K., Danon, L., and Scanlon, T.: Unified Scaling Law for Earthquakes, Phys. Rev. Lett., 88, 178501, doi: 10.1103/PhysRevLett.88.178501, 2002.

Bi, H., Boerner, G., and Chu, Y.: Correlations in the absorption lines of the quasar Q0420-388, Astron. Astrophys. Letters, 218, 19-23, 1989.

Carbone, V., Sorriso-Valvo, L., Harabaglia, P., and Guerra, I.: Unified scaling law for waiting times between seismic events, Europhys. Lett., 71, 1036-1042, doi:10.1209/epl/i2005-10185-0, 2005.

Carbone, V., Sorriso-Valvo, L., Vecchio, A., Lepreti, F., Veltri, P., Harabaglia, P., and Guerra, I.: Clustering of Polarity Reversals of the Geomagnetic Field, Phys. Rev. Lett., 96, 128501, doi:10. 1103/PhysRevLett.96.128501, 2006.

Corral, A.: Local distributions and rate fluctuations in a unified scaling law for earthquakes, Phys. Rev. E, 68, 035102, doi:10.1103/PhysRevE.68.035102, 2003.

Corral, A.: Long-Term Clustering, Scaling, and Universality in the Temporal Occurrence of Earthquakes, Phys. Rev. Lett., 92, 108501, doi:10.1103/PhysRevLett.92.108501, 2004.

Davidsen, J. and Goltz, C.: Are seismic waiting time distributions universal?, Geophys. Res. Lett., 31, 21612, doi:10.1029/2004GL020892, 2004.

Godano, C. and Pingue, F.: Multiscaling in earthquakes diffusion, Geophys. Res. Lett., 32, 18302, doi:10.1029/2005GL023104, 2005.

Guerra, I., Harabaglia, P., Gervasi, A., and Rosa, A. B.: The 19981999 Pollino (Southern Apennines, Italy) seismic crisis: tomography of a sequence, Ann. Geophys., 48(6), 995-1007, 2005.

Gutenberg, B. and Richter, C. F.: Frequency of earthquakes in California, Bull. Seismol. Soc. Am., 34, 185, 1944.

Lepreti, F., Carbone, V., and Veltri, P.: Solar Flare Waiting Time Distribution: Varying-Rate Poisson or Lévy Function?, Astrophys. J. Lett., 555, L133-L136, doi:10.1086/323178, 2001.

Lippiello, E., Godano, C., and de Arcangelis, L.: Dynamical Scaling in Branching Models for Seismicity, Phys. Rev. Lett., 98, 098501, doi:10.1103/PhysRevLett.98.098501, 2007.

Omori, F.: J. Coll. Sci. Imp. Tokyo, 7, 11, 1895. 
Saichev, A. and Sornette, D.: Universal Distribution of Interearthquake Times Explained, Phys. Rev. Lett., 97, 078501, doi:10.1103/PhysRevLett.97.078501, 2006.

Shcherbakov, R., Turcotte, D. L., and Rundle, J. B.: A generalized Omoris law for earthquake aftershock decay, Geophys. Res. Lett., 31, 11613, doi:10.1029/2004GL019808, 2004.

Shcherbakov, R., Yakovlev, G., Turcotte, D. L., and Rundle, J. B.: Model for the Distribution of Aftershock Interoccurrence Times, Phys. Rev. Lett., 95, 218501, doi:10.1103/PhysRevLett.95. 218501, 2005.
Sornette, D.: Earthquakes: from chemical alteration to mechanical rupture, physics report, 313, 237-292, 1999.

Sorriso-Valvo, L., Stefani, F., Carbone, V., Nigro, G., Lepreti, F., Vecchio, A., and Veltri, P.: A statistical analysis of polarity reversals of the geomagnetic field, Phys. Earth Planet. In., 164, 197-207, doi:10.1016/j.pepi.2007.07.001, 2007.

Tosi, P., De Rubeis, V., Loreto, V., and Pietronero, L.: Space-time combined correlation integral and earthquake interactions, Ann. Geophys., 47, 18 491, 854, 2004.

Turcotte, D. L.: Fractals and Chaos in Geology and Geophysics, Cambridge University Press, Cambridge, England, 1993. 